\title{
Expanding the Spectrum of Neurological Manifestations in Cutis Laxa, Autosomal Recessive, Type IIIA
}

\author{
Chloé Angelini ${ }^{1,2}$ Marie Thibaud ${ }^{3}$ Nathalie Aladjidi ${ }^{4,5}$ Pierre Bessou $^{6}$ Sébastien Cabasson ${ }^{3}$ \\ Cindy Colson ${ }^{7}$ Caroline Espil-Taris $^{3} \quad$ Cyril Goizet $^{1,2}$ Marie Husson ${ }^{3}$ Fanny Morice-Picard ${ }^{8}$ \\ Annachiara De Sandre-Giovannoli9,10 Jean-Michel Pédespan ${ }^{3}$
}

\footnotetext{
${ }^{1}$ Service de Génétique Médicale, CHU de Bordeaux and Laboratoire MRGM, INSERM U1211, University of Bordeaux, Bordeaux, France

${ }^{2}$ Centre de Référence Neurogénétique, Service de Génétique Médicale, CHU de Bordeaux, Bordeaux, France

${ }^{3}$ Service de Neurologie Pédiatrique, CHU de Bordeaux, Bordeaux, France

4 Pediatric Hematology Unit, CIC1401, INSERM CICP, University Hospital of Bordeaux, Bordeaux, France

${ }^{5}$ Centre de référence national des cytopénies auto-immunes de I'enfant (CEREVANCE), University Hospital of Bordeaux, Bordeaux, France

${ }^{6}$ Service de Radiologie Pédiatrique, CHU de Bordeaux, Bordeaux, France
}

Address for correspondence Chloé Angelini, MD, Service de Génétique Médicale, CHU de Bordeaux and Laboratoire MRGM, INSERM U1211, University of Bordeaux, Bordeaux, France (e-mail: chloe.angelini@icloud.com).

${ }^{7}$ Normandie Univ, UNICAEN, CHU de Caen Normandie, Department of Genetics, EA7450 BioTARGen, Caen, France.

${ }^{8}$ Centre de Référence des Maladies Rares de la Peau, Service de Dermatologie Pédiatrique, CHU de Bordeaux, Bordeaux, France

${ }^{9}$ Service de Génétique Médicale, Hôpital La Timone Enfants, AP-HM, Marseille, France

${ }^{10}$ CRB-TAC, Centre de ressources biologiques de l'AP-HM, Hôpital La Timone Enfants, AP-HM, Marseille, France

Neuropediatrics 2020;51:245-250.

\begin{abstract}
Keywords

- cutis laxa

- ALDH18A1

- neurological findings

- vascular tortuosity

Cutis laxa is a heterogeneous group of diseases, characterized by abundant and wrinkled skin and a variable degree of intellectual disability. Cutis laxa, autosomal recessive, type IIIA and autosomal dominant 3 syndromes are caused by autosomal recessive or de novo pathogenic variants in ALDH18A1. Autosomal recessive variants are known to lead to the most severe neurological phenotype, and very few patients have been described.

We describe a 13-month-old patient with cutis laxa, autosomal recessive, type IIIA, with an extremely severe phenotype, including novel neurological findings. This description enlarges the neurological spectrum associated to cutis laxa, autosomal recessive, type IIIA, and provides an additional description of this syndrome.
\end{abstract}

\section{Introduction}

Cutis laxa represents a spectrum of clinical entities, including lax and hyperelastic skin with visible veins. It is caused by defective elastin synthesis, structural abnormalities of the extracellular matrix, and also defects in metabolic pathways. These diseases may be acquired or inherited. Inherited cutis laxa are very rare diseases and show different inheritance patterns (autosomal dominant, autosomal recessive, and $\mathrm{X}$-linked recessive inheritance). ${ }^{1}$ To date, 13

received

July 11, 2019

accepted after revision

December 28, 2019

published online

March 6, 2020 different forms of inherited cutis laxa syndromes have been described. $^{2}$

Cutis laxa type IIB (OMIM\#612940), type IIIA (OMIM\#219150), and type IIIB (OMIM\#614438), are known as De Barsy syndrome, and are associated with pathogenic variants in PYCR1 and ALDH18A1, two genes playing an important role in proline metabolism. ${ }^{3}$

PYCR1 encodes for pyrroline-5-carboxylate reductase, an enzyme involved in the last steps of proline synthesis from glutamate. ${ }^{4}$ PYCR1 pathogenic variants can lead to cutis laxa,
DOI https://doi.org/ 10.1055/s-0040-1701671. ISSN 0174-304X. (c) 2020 Georg Thieme Verlag KC Stuttgart · New York 
autosomal recessive, type IIB and type IIIB, with a phenotypic overlap, and are characterized by a cutis laxa of variable severity, abnormal growth, developmental delay, and associated skeletal abnormalities.

ALDH18A1 encodes for delta-1-pyrroline-5-carboxylate synthase (P5CS), an enzyme that catalyzes the reduction of glutamate to delta1-pyrroline-5-carboxylate, and plays a key role in the de novo biosynthesis of proline, ornithine, and arginine. ${ }^{5}$ ALDH18A1 pathogenic variants are responsible for cutis laxa, autosomal recessive, type IIIA, cutis laxa autosomal dominant 3 (OMIM\#616603), and also spastic paraplegia 9A, autosomal dominant (OMIM\#601162), and 9B, autosomal recessive (OMIM\#616586). There is a phenotypic overlap between these four syndromes, with variable neurologic symptoms.

Cutis laxa, autosomal recessive, type IIIA, is a rare genetic condition. ${ }^{6,7}$ Only about 20 patients have been described in the literature. ${ }^{8}$ Its clinical spectrum includes abundant and wrinkled skin, skeletal anomalies, neurodevelopmental disorders, cataract and corneal clouding, and, in some cases, severe neurological symptoms such as major developmental delay, hypotonia in infancy, hyperreflexia, athetoid movements, and seizures. ${ }^{9,10}$ The cellular phenotype is characterized by diminished production of collagen types I and III, altered elastin ultrastructure, and diminished cell proliferation. ${ }^{11}$

Cutis laxa, autosomal dominant 3 , is characterized by excessive skin with visible veins, cataract or corneal clouding, clenched fingers, pre- and postnatal growth retardation, and moderate intellectual disability. Some patients may also present muscular hypotonia with brisk muscle reflexes and cranial vessel tortuosity with foramen magnum stenosis. The phenotype seems to be less severe than cutis laxa, autosomal recessive, type IIIA, but with a greater neurological involvement, with hypertonia, spasticity, and brain magnetic resonance imaging (MRI) abnormalities, such as tortuous intracranial vessels, widened perivascular spaces, and white matter signal anomalies on T2 and fluid-attenuated inversion recovery (FLAIR) sequences. ${ }^{12}$

Spastic paraplegia 9A is a neurologic disorder with progressive spasticity appearing from adolescence to adulthood. Patients present with lower limbs spasticity, and may have additional features, such as cataract, cerebellar signs, and urinary urgency. ${ }^{4}$

Spastic paraplegia 9B tends to be more severe, with childhood onset spastic paraplegia, delayed psychomotor development, intellectual disability, and severe motor impairment. ${ }^{4}$

We describe here a 13-month-old patient, with a homozygous missense pathogenic variant in ALDH18A1. He had a very severe phenotype, with strong neurological involvement. This description widens the phenotypic spectrum associated with mutations of this gene.

\section{Clinical Report}

The patient was the first child of a consanguineous couple of first cousins, who were both from Senegal. No medical relevant history was found in their families.
During pregnancy appeared severe intrauterine growth retardation (IUGR), associated to oligoamnios. Amniocentesis was done to perform a genetic work up, including an array-comparative genomic hybridization and a karyotype, both were normal.

He was born at 34 gestational weeks by a cesarean section, planned because of IUGR. He needed breathing assistance immediately after birth, with noninvasive ventilation, during 3 hours. He weighed $1.66 \mathrm{~kg}$ ( -3.9 standard deviation [SD]), measured $40 \mathrm{~cm}(-5 \mathrm{SD})$, and his head circumference was $31 \mathrm{~cm}$ (-3.8 SD).

Examination at birth revealed a large fontanel, adductus thumbs, global hypotonia, and a major systolic cardiac murmur. Transfontanellar ultrasound was normal, as well as an electroencephalogram, an abdominal ultrasound, and skeletal X-rays. Cardiac ultrasound found a large ventricular septal defect and a large atrial septal defect. Ophthalmological examination with eye fundoscopy revealed bilateral corneal clouding.

Neurological examination worsened with months and he presented with four limbs spasticity, permanent tremor, and eye twitching. He had also severe dystonia and four limbs contractures that led to hip and shoulder dislocations (-Fig. 1). Adductus thumbs as well as calcaneus valgus feet were persistent.

He presented at age 5 months partial seizures with focal onset and motor manifestations (focal myoclonia), recorded on electroencephalogram with multifocal spikes, and slow waves with very slow background activity and absence of sleep spindles (-Fig. 2). He was from then effectively treated with oxcarbazepine.

A brain MRI was performed at age 8 months (-Fig. 3 ). Diffuse supra- and infratentorial cortical atrophy was found, with brain stem contours distortions, associated to a thin corpus callosum and large ventricles due to gray matter paucity. A severe dysmyelination was observed with abnormal spectroscopy (lactate increased and $\mathrm{N}$-acetyl aspartate decreased). Basal ganglia were hardly identified. Willis polygon vessels were abnormal, and described as megadolichovessels.

He exhibited a severe failure to thrive during follow-up. He presented with multiple regurgitations and feeding was performed with a nasogastric tube. At age 13 months, he weighed $3.46 \mathrm{~kg}(-6.0 \mathrm{SD})$, measured $50 \mathrm{~cm}(-10.1 \mathrm{SD})$, and head circumference was $37 \mathrm{~cm}$ (-8.2 SD).

His skin was very wrinkled and abundant. He had three episodes of skin abscesses, with persistent axillar adenopathies. He also had several episodes of respiratory distress without opportunist infections. Humoral and cellular immune analyses were normal. Blood lactate assay was normal at $1.5 \mathrm{mmol} / \mathrm{L}$.

Dysmorphia was marked, with a triangular face, microcephaly, small mouth, retrognathism, and posteriorly rotated ears (-Fig. 1).

He died at age 16 months, after 2 weeks of resopiratory infection and global worsening of his general state. 

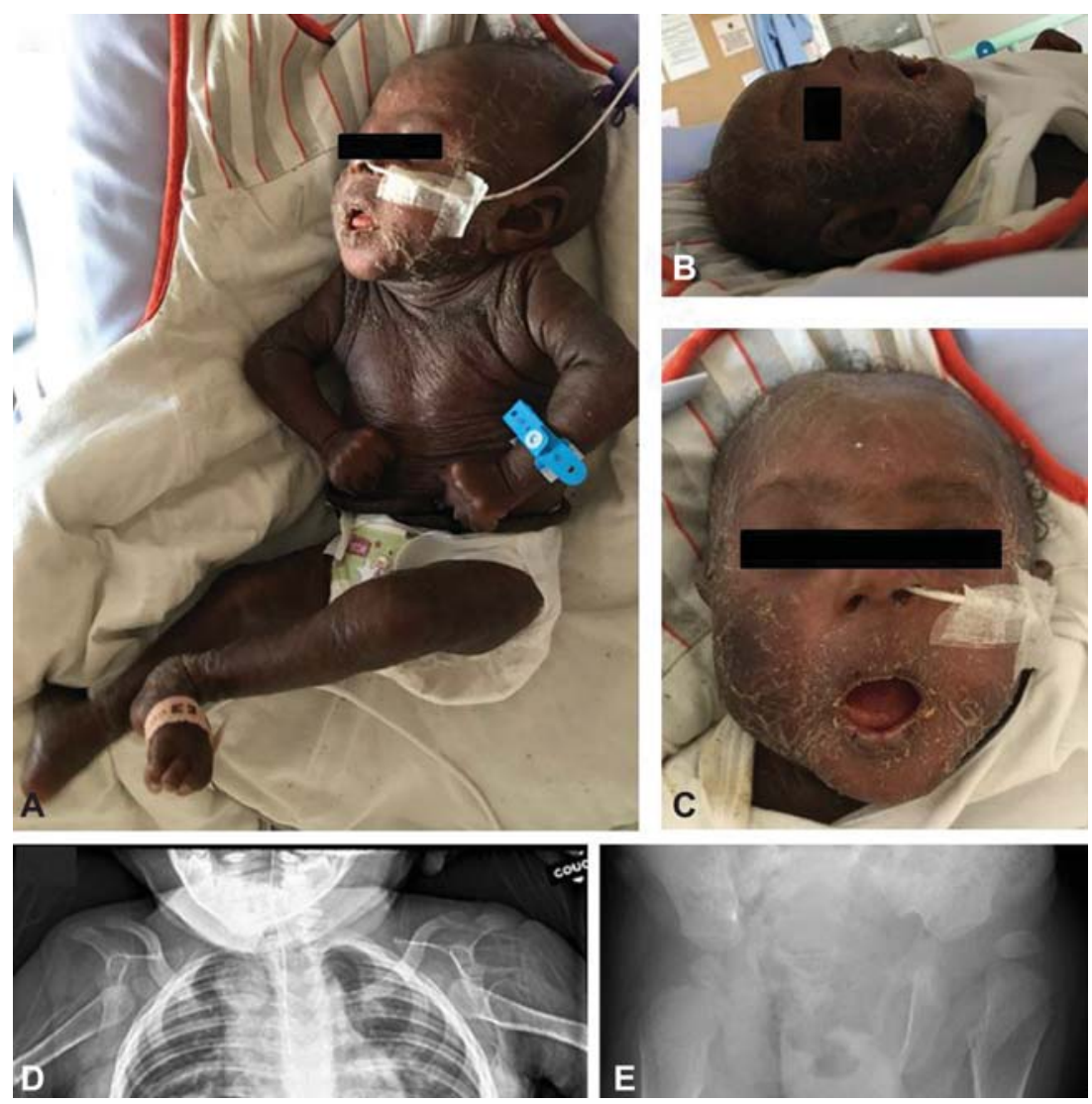

Fig. 1 Clinical presentations: photographs aged 11 months (A, B, C) and X-rays (D: chest X-rays and E: pelvis X-rays).Observe the dysmorphia with triangular face, low and posteriorly rotated ears, anteverted nares, and small mouth with thin vermilion lips. Skin is wrinkled, abundant, and dry with cracks on the face. Note four limb contractures with dislocated shoulders and hips, also present on X-rays.

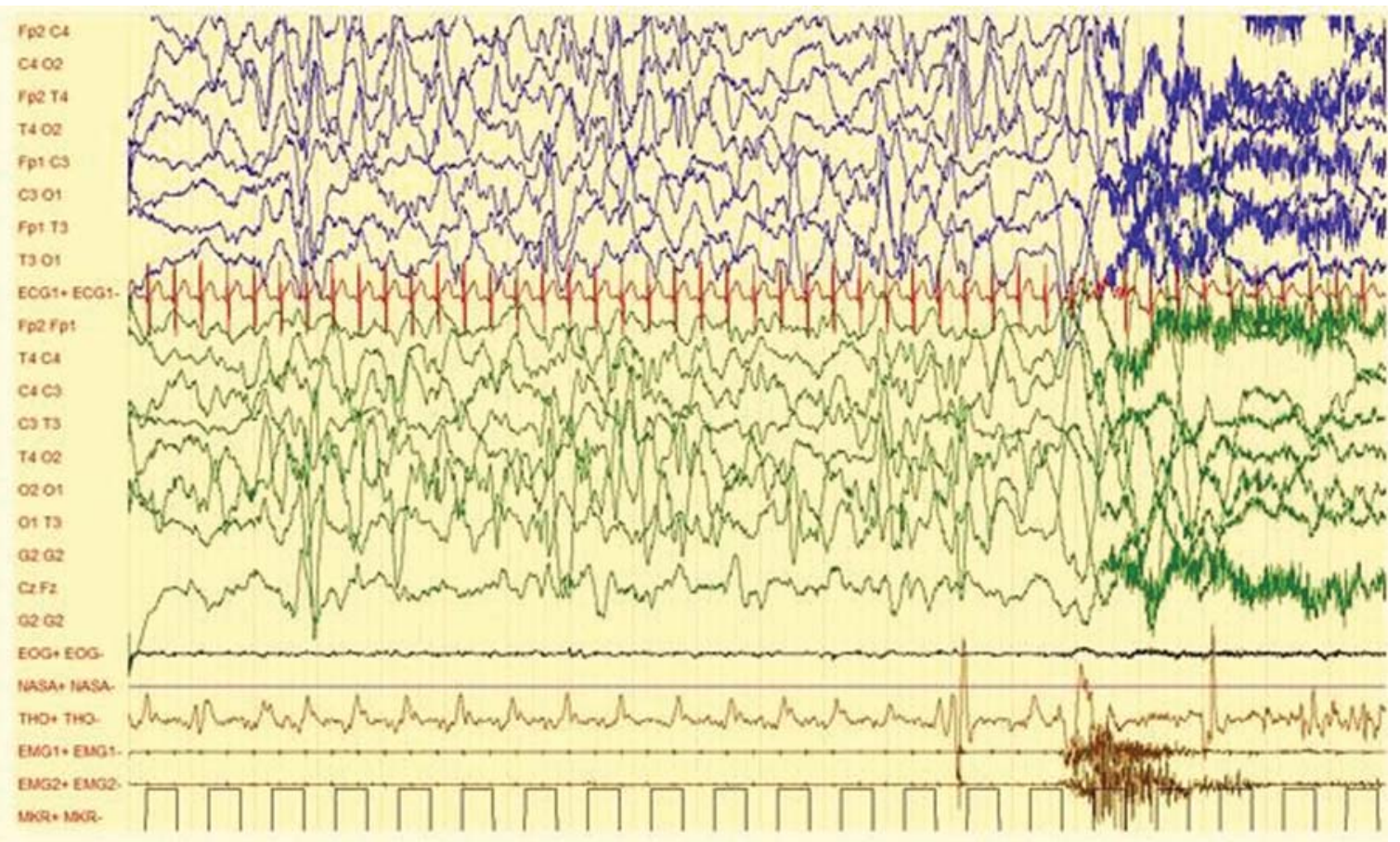

Fig. 2 Electroencephalogram data. Standard parameters, $70 \mathrm{~Hz}, 70 \mu \mathrm{V} / \mathrm{cm}$. Sleep interictal electroencephalogram showed active and irregular discharge of multifocal spikes, slow waves with abnormal (very slow) background activity, and absence of sleep spindles. No cluster of spasms were recorded after a focal seizure. 

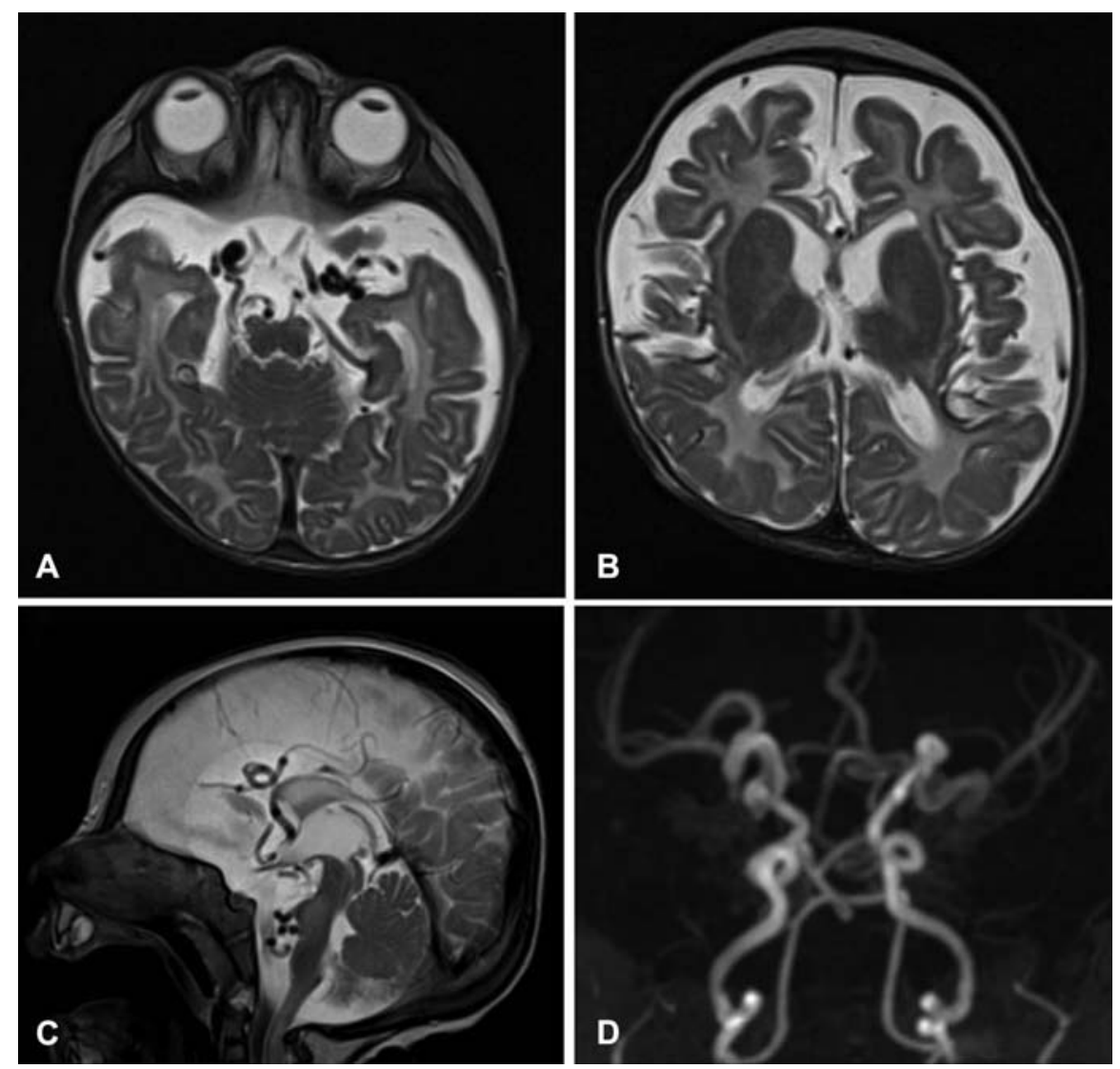

Fig. 3 Brain magnetic resonance imaging (MRI). Axial T2-weighed sequences (A and B), sagittal T2-weighed sequences (C) and threedimensional (3D) time-of-flight (TOF) (D). Observe the supra- and infratentorial cortical atrophy and the thin corpus callosum. Dysmyelination can be observed in B. Interesting features to note are megadolichovessels of Willis polygone (A, C, D).

\section{Molecular Analysis}

Deoxyribonucleic acid was extracted from peripheral blood samples of the patient and his parents. The targeted nextgeneration sequence analysis of a 82 genes panel including genes involved in premature aging and laminopathies was performed for the proband (gene list available on orphanet, https://www.orpha.net/consor/cgi-bin/ClinicalLabs_Search. php?lng=FR\&data_id=128241\&search=ClinicalLabs_Search_ Simple\&data_type=Test\&title=Diagnostic\%20des\%20laminopathies\%20et\%20du\%20vieillissement\%20prematur\%DA\%20 Panel\&MISSING\%20CONTENT=Diagnostic-des-laminopathies-et-du-vieillissement-prematur-Panel-).

A SureSelectXT 12 to $24 \mathrm{Mb}$ library (Agilent Technologies, Santa Clara, California, United States) was used for enrichment of targeted sequences using the SureSelect Target Enrichment System from Agilent. The Ion Proton platform (Thermo Fisher Scientific, United States) was used for highthroughput sequencing. Then, raw data were converted to Fastq files and aligned to the reference sequence of the human genome (University of California Santa Cruz, hg19/ GRCh37), using the Torrent Suite software (Thermo Fisher).

A variant calling format file and binary alignment map and index files were then obtained and used for variant annotation using the in-house software VarAFT (Variant Annotation and Filtration Tool). ${ }^{13}$ Sequence reads and variants' visualization was performed using IGV (Integrative Genomics
Viewer). ${ }^{14}$ The variants were classified according to the American College of Medical Genetics and Genomics classification. ${ }^{15}$

This targeted next-generation sequencing led to the identification of a homozygous pathogenic variant in ALDH18A1, NM_002860.3: exon 13: c.1499G > T, p. (Gly500Val) (ClinVar VCV000638645.1). This variant was absent from Human Gene Mutation Database (HGMD), GnomAD, ExAC, and 1000 Genomes databases. All of the in silico prediction programs, including Mutation Taster, UMD-Predictor, and Human Splicing Finder predicted this variant to be deleterious. Combined Annotation Dependent Depletion score was assessed at 27.8, a high score, supporting predictions of the programs. This variant was classified as likely pathogenic (PM1, PM2, PM3, PP3) according to the American College of Medical Genetics and Genomics classification. ${ }^{15}$

Sanger sequencing of ALDH18A1 exon 13 proofed this variant in a homozygous state in the index patient and revealed it in a heterozygous state in the parents, consistent with autosomal recessive inheritance.

\section{Discussion}

We present a patient with a cutis laxa, autosomal recessive, type IIIA. This syndrome is rare with only about 20 patients described to date. 
His clinical phenotype is similar to the other cases described. ${ }^{8}$ However, few data are available regarding neuroimaging. Only eight patients with autosomal recessive variants underwent a brain MRI, which highlighted white matter anomalies for most of patients, a thin corpus callosum in three, and vascular tortuosity in one. ${ }^{3,16}$ One of the patients described had a normal brain MRI. ${ }^{17}$

De novo ALDH18A1 variants lead to a cutis laxa, autosomal dominant 3 syndrome, with a milder phenotype, but with a higher risk of developing hypertonia and spasticity. ${ }^{12} \mathrm{~A}$ recent review from Sinnige et al describes three patients with de novo variants in ALDH18A1, and sums up the different neuroradiological findings in the literature. ${ }^{18}$ Eleven patients are reported with dominant negative variants, and the main findings were tortuous intracranial vessels, widened perivascular spaces, and white matter signal anomalies on T2 and FLAIR sequences.

In our patient, tortuous intracranial vessels were noted and were predominant on Willis polygon circle. This is concordant with the description in the Sinnige et al review, where tortuous vessels were present mostly on arteries, only two patients were described with venous tortuosity, and only on the cerebral and basilar arteries. ${ }^{18}$ Martinelli et al hypothesized that this tortuosity could be secondary to abnormal proline metabolism, affecting the production of proline-rich connective tissue proteins such as elastin and collagen and then leading to defective interactions between the extracellular matrix and vascular smooth muscle. ${ }^{19}$

The data on our patient enlarge the spectrum of neuroimaging findings and provides an additional global description of a patient with cutis laxa, autosomal recessive, type IIIA. A link is thus established between the neurological phenotypes of autosomal dominant 3 and recessive type IIIA cutis laxa syndromes.

Moreover, this variant has never been described before. We performed an in silico analysis to establish its pathogenicity and also provide an explanation of the patient's clinical symptoms, which belong both to autosomal recessive type IIIA and autosomal dominant 3 cutis laxa.

P5CS, the enzyme encoded by this gene, is a single-chain homo-oligomeric protein, and is composed of two main domains, glutamate 5-kinase (G5K) and glutamyl-5-phosphate reductase (G5PR). ${ }^{5,20}$ The G5PR domain is divided into four parts: a cofactor binding domain, a catalytic domain, and two oligomerization domains. The oligomerization domains are close one to another in a tertiary structure, and the cofactor binding and catalytic domains interact with another P5CS in a quaternary structure, thanks to inter-subunit salt bridges.

No hotspot of pathogenic variants has been described for cutis laxa autosomal recessive type IIIA, as attested by the Clinvar and HGMD. For cutis laxa autosomal dominant 3, a predominance of a missense variant on residue Arg138 has been observed. ${ }^{12}$ Mainly missense variants are described for the four pathologies linked to ALDH18A1. The position of our variant, p.Gly500, is considered as intolerant to missense variations in the Metadome database (score at 0.38). ${ }^{21}$
Our variant is located in the cofactor binding domain of G5PR, and in quaternary structure sits at the junction of the cofactor binding domain, the catalytic domain, and the oligomerization domain of the G5PR part of the protein, as shown in Magini et al publication. ${ }^{20}$ We hypothesize that the amino acid change due to our variant disturbs the global equilibrium of the oligomerization.

However, these in silico analyses cannot account fully for the pathogenicity of the patient's variant, or explain the phenotypic overlap between autosomal recessive and dominant cutis laxa. While the patient's parents are symptomfree, it would have been interesting to perform functional studies in skin biopsies of the patient and his parents. Evaluation of proline biosynthesis after loading ${ }^{13} \mathrm{C}_{5}$-labeled glutamine, and comparison of the flux between controls, parents, and patient would have provided interesting data. It may have shown a significant reduction in proline biosynthesis in the patient, consistent with an enzymatic deficiency of P5CS. ${ }^{4}$ Results for the parents might be harder to interpret as they are symptom-free, but a slight decrease in flux could be interpreted as a partial dominant negative effect of the variants.

In conclusion, this work suggests the phenotypic overlap between the four syndromes due to ALDH18A1 pathogenic variants, and underscores the need to perform a brain MRI in every patient with ALDH18A1-related disorders, whatever the type of variants highlighted.

Conflict of Interest

None declared.

\section{References}

1 Mohamed M, Voet M, Gardeitchik T, Morava E. Cutis laxa. In: Halper J, ed. Progress in Heritable Soft Connective Tissue Diseases [Internet]. Dordrecht: Springer Netherlands; 2014:161-184. (Advances in Experimental Medicine and Biology). Available at: https://doi.org/10.1007/978-94-007-7893-1_11. Accessed September 26, 2019

2 Ritelli M, Cammarata-Scalisi F, Cinquina V, Colombi M. Clinical and molecular characterization of an 18-month-old infant with autosomal recessive cutis laxa type $1 \mathrm{C}$ due to a novel LTBP4 pathogenic variant, and literature review. Mol Genet Genomic Med 2019;7(07):e00735

3 Wolthuis DFGJ, van Asbeck E, Mohamed M, et al. Cutis laxa, fat pads and retinopathy due to ALDH18A1 mutation and review of the literature. Eur J Paediatr Neurol 2014;18(04):511-515

4 Coutelier M, Goizet C, Durr A, et al. Alteration of ornithine metabolism leads to dominant and recessive hereditary spastic paraplegia. Brain 2015;138(Pt 8):2191-2205

5 Panza E, Escamilla-Honrubia JM, Marco-Marín C, et al. ALDH18A1 gene mutations cause dominant spastic paraplegia SPG9: loss of function effect and plausibility of a dominant negative mechanism. Brain 2016;139(Pt 1):e3-e3

6 Kivuva EC, Parker MJ, Cohen MC, Wagner BE, Sobey G. De Barsy syndrome: a review of the phenotype. Clin Dysmorphol 2008;17 (02):99-107

7 Bicknell LS, Pitt J, Aftimos S, Ramadas R, Maw MA, Robertson SP. A missense mutation in ALDH18A1, encoding Delta1-pyrroline-5carboxylate synthase (P5CS), causes an autosomal recessive neurocutaneous syndrome. Eur J Hum Genet 2008;16(10):1176-1186

8 Lefebvre M, Beaufrere A-M, Francannet C, et al. Extending the ALDH18A1 clinical spectrum to severe autosomal recessive fetal 
cutis laxa with corpus callosum agenesis. Am J Med Genet A 2018; 176(11):2509-2512

9 de Barsy AM, Moens E, Dierckx L. Dwarfism, oligophrenia and degeneration of the elastic tissue in skin and cornea. A new syndrome? Helv Paediatr Acta 1968;23(03):305-313

10 Hoefnagel D, Pomeroy J, Wurster D, Saxon A. Congenital athetosis, mental deficiency, dwarfism and laxity of skin and ligaments. Helv Paediatr Acta 1971;26(04):397-402

11 Further expansion of the phenotypic spectrum associated with mutations in ALDH18A1, encoding $\Delta 1$-pyrroline-5-carboxylate synthase (P5CS) - Skidmore - 2011 - American Journal of Medical Genetics Part A - Wiley Online Library [Internet]. Available at: https://onlinelibrary.wiley.com/doi/epdf/10.1002/ajmg.a.34057. Accessed June 4, 2019

12 Fischer-Zirnsak B, Escande-Beillard N, Ganesh J, et al. Recurrent de novo mutations affecting residue Arg138 of pyrroline-5-carboxylate synthase cause a progeroid form of autosomal-dominant cutis laxa. Am J Hum Genet 2015;97(03):483-492

13 Desvignes J-P, Bartoli M, Delague V, et al. VarAFT: a variant annotation and filtration system for human next generation sequencing data. Nucleic Acids Res 2018;46(W1):W545-W553

14 Robinson JT, Thorvaldsdóttir H, Winckler W, et al. Integrative genomics viewer. Nat Biotechnol 2011;29(01):24-26

15 Richards S, Aziz N, Bale S, et al; ACMG Laboratory Quality Assurance Committee. Standards and guidelines for the interpretation of sequence variants: a joint consensus recommendation of the American College of Medical Genetics and Genomics and the Association for Molecular Pathology. Genet Med 2015;17(05):405-424
16 Baumgartner MR, Rabier D, Nassogne M-C, et al. $\Delta$ 1-pyrroline-5carboxylate synthase deficiency: neurodegeneration, cataracts and connective tissue manifestations combined with hyperammonaemia and reduced ornithine, citrulline, arginine and proline. Eur J Pediatr 2005;164(01):31-36

17 Fischer B, Callewaert B, Schröter P, et al. Severe congenital cutis laxa with cardiovascular manifestations due to homozygous deletions in ALDH18A1. Mol Genet Metab 2014;112(04): 310-316

18 Sinnige PF, van Ravenswaaij-Arts CMA, Caruso P, et al. Imaging in cutis laxa syndrome caused by a dominant negative ALDH18A1 mutation, with hypotheses for intracranial vascular tortuosity and wide perivascular spaces. Eur J Paediatr Neurol 2017;21(06): 912-920

19 Martinelli D, Häberle J, Rubio V, et al. Understanding pyrroline5-carboxylate synthetase deficiency: clinical, molecular, functional, and expression studies, structure-based analysis, and novel therapy with arginine. J Inherit Metab Dis 2012;35(05): 761-776

20 Magini P, Marco-Marin C, Escamilla-Honrubia JM, et al. P5CS expression study in a new family with ALDH18A1-associated hereditary spastic paraplegia SPG9. Ann Clin Transl Neurol 2019;6(08):1533-1540

21 MetaDome. Pathogenicity analysis of genetic variants through aggregation of homologous human protein domains - Wiel - 2019 - Human Mutation - Wiley Online Library [Internet]. Available at: https://onlinelibrary.wiley.com/doi/full/10.1002/humu.23798. Accessed October 8, 2019 